Marco Lunanova

Optimierung von Nebenaggregaten 


\section{VIEWEG+TEUBNER RESEARCH}


Marco Lunanova

\section{Optimierung von \\ Nebenaggregaten}

Maßnahmen zur Senkung der $\mathrm{CO}_{2}$-Emission von Kraftfahrzeugen 
Bibliografische Information der Deutschen Nationalbibliothek

Die Deutsche Nationalbibliothek verzeichnet diese Publikation in der

Deutschen Nationalbibliografie; detaillierte bibliografische Daten sind im Internet über

<http://dnb.d-nb.de> abrufbar.

1. Auflage 2009

Alle Rechte vorbehalten

(C) Vieweg+Teubner | GWV Fachverlage GmbH, Wiesbaden 2009

Lektorat: Christel A. Roß

Vieweg+Teubner ist Teil der Fachverlagsgruppe Springer Science+Business Media. www.viewegteubner.de

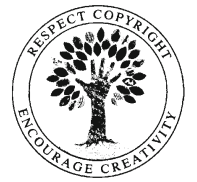

Das Werk einschließlich aller seiner Teile ist urheberrechtlich geschützt. Jede Verwertung außerhalb der engen Grenzen des Urheberrechtsgesetzes ist ohne Zustimmung des Verlags unzulässig und strafbar. Das gilt insbesondere für Vervielfältigungen, Übersetzungen, Mikroverfilmungen und die Einspeicherung und Verarbeitung in elektronischen Systemen.

Die Wiedergabe von Gebrauchsnamen, Handelsnamen, Warenbezeichnungen usw. in diesem Werk berechtigt auch ohne besondere Kennzeichnung nicht zu der Annahme, dass solche Namen im Sinne der Warenzeichen- und Markenschutz-Gesetzgebung als frei zu betrachten wären und daher von jedermann benutzt werden dürften.

Umschlaggestaltung: KünkelLopka Medienentwicklung, Heidelberg

Druck und buchbinderische Verarbeitung: STRAUSS GMBH, Mörlenbach

Gedruckt auf säurefreiem und chlorfrei gebleichtem Papier.

Printed in Germany

ISBN 978-3-8348-0730-4 


\section{Inhaltsverzeichnis}

$1 \quad$ Einleitung und Zieldefinition................................................1

$2 \quad \mathrm{CO}_{2}$-Emissionen.................................................................

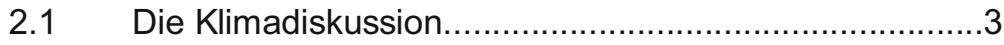

$2.2 \quad$ Anthropogene Treibhausgase ......................................

$3 \quad$ Stand der Technik.............................................................

3.1 Auslegungskriterien von Nebenaggregaten und Leistungsvariable im Betrieb...................................................

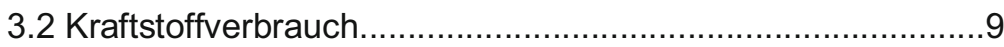

3.3 NEFZ vs. kundenrelevantes Fahrprofil................................15

3.4 Einfluss der Nebenaggregate auf den Kraftstoffverbrauch...20

3.4.1 Übersicht verbrauchsrelevanter Nebenaggregate20

3.4.2 Kühlmittelpumpe $\ldots \ldots \ldots \ldots \ldots \ldots \ldots \ldots \ldots \ldots \ldots \ldots \ldots \ldots \ldots \ldots \ldots \ldots \ldots . .24$

3.4.3 Lüfter...............................................................32

3.4.4 Lenkungssysteme...............................................

3.4.4.1 Manuelle Lenkung...................................43

3.4.4.2 Hydraulische Lenkung...........................43

3.4.4.3 Elektro-hydraulische Lenkung (EHPS).. 49

3.4.4.4 Elektrische Lenkung (EPS).....................55

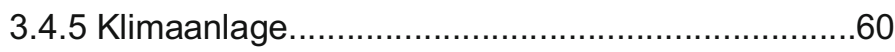


3.4.6 Vakuumpumpe............................................ 71

3.4.7 Luftpresser................................................ 72

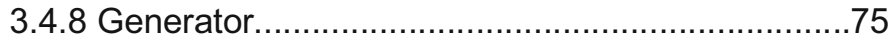

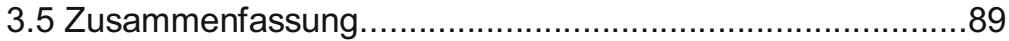

$4 \quad$ Optimierung von Nebenaggregaten....................................93

4.1 Ergebnisse vorangegangener Arbeiten............................94

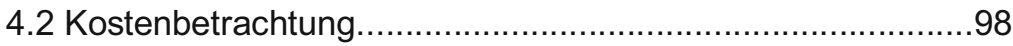

4.3 Optimierung des Kühlsystems.......................................99

4.3.1 Thermomanagement......................................100

4.3.2 Modifizierte Kühlmittelpumpen.........................105

4.3.2.1 Elektrische Kühlmittelpumpen.............105

4.3.2.2 Kühlmittelpumpenantrieb....................112

4.3.2.3 Konventionelle KühImittelpumpen.......122

4.3.3 Modifizierte Lüfter............................................124

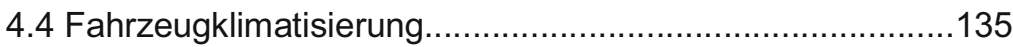

4.4.1 Reduzierung der Antriebsleistung.....................136

4.4.1.1 Klimakompressor.............................136

4.4.1.2 Kältekreislauf..................................163

4.4.1.3 Bedarfsgerechte Klimatisierung durch Enthalpie Regelung............................169

4.4.1.4 Kompressorantrieb..........................176 
4.5 Lenkungssysteme.

4.5.1 EHPS - Systeme für Nutzfahrzeuge.

4.5.2 Volumenstromgeregelte Hydraulikpumpe 200

4.5.3 Pumpenantrieb 205

4.5.4 Hydrauliklenkung mit Zusatzsystem .206

4.6 Generator 217

4.7 Sonstige Nebenaggregate. .236

4.7.1 Vakuumpumpe .236

4.7.2 Luftpresser 240

4.8 Optimierter Antrieb von Nebenaggregaten im Verbund......245 4.9 Zusammenfassung. 260

5 Sinnvolle Kombination von Optimierungsmaßnahmen. .267

6 Schlussfolgerung und Ausblick. .275

7 Literaturverzeichnis. .279

8 Abbildungsverzeichnis. .287

9 Tabellenverzeichnis. .301 


\section{Verwendete Formelzeichen und ihre Einheiten}

Die nachfolgenden Bezeichnungen, Abkürzungen und Indizes werden nach Möglichkeit grundsätzlich verwendet, wobei Abweichungen und Ergänzungen von diesen Formelzeichen jeweils bei den entsprechenden Gleichungen oder Abbildungen genannt werden.

\section{Zeichen}

$P$

$\mathrm{t}$

E

A

$\mathrm{CO}_{2}$

$\mathrm{h}$

$b_{e}$

B

$\mathrm{V}$

W

Q

[ ]

( )

$\mathrm{H}_{\mathrm{u}}$

M

$\mathrm{n}$

$\mathrm{K}$

$\mathrm{B}_{0}$

C

$\mathrm{v}$

$\mathrm{p}$

$\mathrm{MPa}$

w

Z

$\dot{V}$

I

U
Bedeutung

Leistung

Zeit

Energie

Fläche, Querschnitt

Kohlendioxid

spezifische Enthalpie

spezifischer Kraftstoffverbrauch

(stündlicher) Kraftstoffverbrauch

Volumen

Arbeit

Wärme

Literaturverweis

Gleichung

spezifischer Heizwert

Drehmoment

Drehzahl

Mehrverbrauchsfaktor

Nullleistungsverbrauch

(Mehr)Verbrauch

Geschwindigkeit

Druck

Strömungsgeschwindigkeit

Höhe

Volumenstrom

elektrischer Strom

elektrische Spannung

\section{Einheit}

$\mathrm{W}, \mathrm{kW}$

$\mathrm{s}, \mathrm{h}$

$\mathrm{J}, \mathrm{Nm}$

$\mathrm{mm}^{2}$

$\mathrm{kJ} / \mathrm{kg}$

$\mathrm{g} / \mathrm{kWh}$

$\mathrm{g} / \mathrm{h}, \mathrm{kg} / \mathrm{h}$

L, $\mathrm{cm}^{3}$

$\mathrm{J}, \mathrm{Nm}$

$\mathrm{J}, \mathrm{Nm}$

-

$\mathrm{MJ} / \mathrm{kg}$

$\mathrm{Nm}$

$\min ^{-1}$

l/kWh

$\mathrm{g} / \mathrm{h}, \mathrm{kg} / \mathrm{h}$

l/100 km

$\mathrm{km} / \mathrm{h}$

bar, kPa,

$\mathrm{m} / \mathrm{s}$

$\mathrm{m}$

$\mathrm{m}^{3} / \mathrm{h}$,

$\mathrm{I} / \mathrm{min}$

A

V 


$\begin{array}{lll}\mathrm{T}, \mathrm{t} & \text { Temperatur } & { }^{\circ} \mathrm{K},{ }^{\circ} \mathrm{C} \\ \dot{m} & \text { Massenstrom } & \mathrm{kg} / \mathrm{h} \\ \mathrm{F} & \text { Kraft } & \mathrm{N} \\ \mathrm{i} & \text { Übersetzung } & - \\ \mathrm{e} & \text { Potenzialdifferenz } & \mathrm{V}, \mathrm{m} / \mathrm{s}, \mathrm{rad} / \mathrm{s}, \mathrm{Pa}, \mathrm{J} / \mathrm{kg} \\ \mathrm{f} & \text { Energiefluss } & \mathrm{A}, \mathrm{N}, \mathrm{Nm}, \mathrm{m}^{3} / \mathrm{s}, \mathrm{kg} / \mathrm{s}\end{array}$

Griechische Formelzeichen

Zeichen

$\rho$

$\eta$

$\Delta$

$\varphi$

$\omega$

\section{Indizes (Fußzeiger)}

\section{Index}

e, eff

$\mathrm{zu}$

Otto

Diesel

$\mathrm{h}$

a

e

$\mathrm{P}$

ges

is

vol

mech

rel

ND

$\mathrm{HD}$

$M$

G

\section{Bedeutung}

Dichte

Wirkungsgrad

Differenz

Getriebespreizung

Winkelgeschwindigkeit

\section{Einheit}

$\mathrm{kg} / \mathrm{cm}^{3}$

-

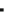

$\mathrm{rad} / \mathrm{s}, \mathrm{s}^{-1}$

\section{Bedeutung}

effektiv

zugeführt

bezogen auf den Ottomotor

bezogen auf den Dieselmotor

hydraulisch

Ausgang

Eingang

Pumpe

gesamt

isentrop

volumetrisch

mechanisch

relativ

Niederdruck

Hochdruck

Motor

Generator 


\section{Konstanten}

\section{Zeichen}

g

$\pi$
Bedeutung

Erdbeschleunigung

Kreiszahl $\underline{\text { Wert }}$

$9,81 \mathrm{~m} / \mathrm{s}^{2}$

3,1415927

\section{Abkürzungen}

EHPS

EPS

$\mathrm{P} / \mathrm{S}$

$\mathrm{A} / \mathrm{C}$

PM

CVT

NEFZ

NEUDC

PKW

NFZ

FCM

PWM

p.a.
Electro-Hydraulic-Power-Steering (Elektro

hydraulische-Lenkung)

Electric Power Steering (Elektrische Lenkung)

Power Steering (konvent. Hydrauliklenkung)

Air conditioning (Klimaanlage)

Permanentmagnet

Continously Variable Transmission (stufenloses

Getriebe)

Neuer Europäischer Fahrzyklus

New European Driving Cycle (= NEFZ)

Personenkraftwagen

Nutzfahrzeug

Fan Control Module (Lüfterregelung)

Pulsweitenmodulation

per anno (pro Jahr) 